\title{
Standounkt
}

Wissenschaft muss von den Problemen her denken

\section{Das Prachtschloss Interdisziplinarität}

\author{
Große Themen wie Klimawandel und Gesundheit lassen sich \\ nur trans- und interdisziplinär lösen. Doch die Wissenschafts- \\ community sträubt sich gegen fächerübergreifende Zusammen- \\ arbeit. Auch Forschungseinrichtungen und -förderer müssen \\ umdenken. Von Claus Leggewie
}

$I^{\text {mon }}$ m Wissenschaftsjahr 2012 war viel und lobend von Interdisziplinarität die Rede. Viele Fächer, die heute ein gesichertes Methodenrepertoire und Grenzen gegenüber anderen Disziplinen beanspruchen, sind selbst aus der Fusion von Wissensbeständen in Einzelfächern hervorgegangen. So gesehen ist eher die institutionelle Absonderung erklärungsbedürftig; wissenschaftlicher Fortschritt wird erfahrungsgemäß eher an den Rändern als im Herz der Fächer produziert. Kooperation sollte in einer Einrichtung, die sich Universitas nennt, eigentlich die Regel sein.

Das Gros der Probleme, mit denen wir es heute zu tun haben, sprengt die Grenzen eines Faches. Dass man offene Fragen wie die Gesundheitsversorgung armer oder alternder Gesellschaften oder die Folgen von Klimawandel lösen soll, mag manchen Spezialisten als Eingriff in die Wissenschaftsfreiheit vorkommen, aber was "Gesundheit" oder „Klima“ überhaupt ist, muss auch für sie ein Erkenntnisproblem darstellen, für das eben nicht ein Fach, die Medizin oder die Meteorologie, zuständig ist.

Hier bietet sich der weiterführende Terminus Transdisziplinarität an, den Jürgen Mittelstraß eingeführt hat; sie besitzt keine eigenständige Methodik, sondern ist als ein Forschungs- und Wissenschaftsprinzip zu betrachten. Gefragt ist heute eine "disziplinierte Interdisziplinarität in transdisziplinären Prozessen“, die Roland W. Schulz im Blick auf die Mensch-Natur-Interaktion, Uwe Schnei- dewind für die Nachhaltigkeitsthematik und der Wissenschaftliche Beirat Globale Umweltfragen (WBGU) für die Transformationsforschung postuliert haben. Für systemische Betrachtungsweisen der Koevolution von Natur, Gesellschaft und Wissenschaft ist dieser Ansatz besonders bedeutsam.

Er beinhaltet im Übrigen den gemeinsamen Lernprozess von Fachmenschen und Laien auf Augenhöhe, gerade wo es um Risiken geht. Vor diesem Realitätstest schrecken die meisten Forscher(innen) zurück. Forschung muss aber nicht nur den Kontakt zu Unternehmen und Verwaltungen halten, sondern auch zu den Change Agents, den Pionieren des Wandels in der Bürgergesellschaft.

Inter- und Transdisziplinarität wird im Wissenschaftssystem nicht wirklich belohnt. Während die Teilgebiete der großen Forschungsgemeinschaften sich rasant spezialisieren, setzen große Förderinstitutionen (Deutsche Forschungsgesellschaft, Bundesministerium für Bildung und Forschung, Stiftung Volkswagen, Stiftung Mercator) Inter- und Transdisziplinarität auf die Tagesordnung. So begrüßenswert das ist, wird es bislang durch die Modalitäten der Begutachtungen im Peer-ReviewVerfahren und die üblichen Karrierewege des Wissenschaftssystems nicht gedeckt. Viele Ansätze scheitern an den Beharrungskräften des in der Wolle prodisziplinär gefärbten Wissenschaftssystems: Gutachter-Fachkollegien hüten misstrauisch ihre Vorgärten, Forschungsrektoren sperren sich gegen fächerübergreifende Stu- diengänge und Arbeitszusammenhänge oder erlauben sie höchstens als freiwillige Zusatzbeschäftigung. Dissertationen und Habilitationen, die sich Problemen der realen Welt zuwenden und dadurch per se transdisziplinär geraten, fallen durch den Rost der Selektionssysteme und gelten an der Basis nicht nur als riskant, sondern als ansehens- und karriereschädlich. Dazu passt die dogmatische Auffassung vieler älterer Fachvertreter, interdisziplinäre Arbeit könne überhaupt erst nach Jahrzehnten erfolgreicher disziplinärer Forschung einsetzen, und die Standard-Organisation von Bachelor-Studiengängen, die Anfänger erst gar nicht auf die Idee kommen lässt, über den Zaun zu blicken, und eine spontane Neugier, die sie vielleicht von gut gemachten Projektwochen aus der Schule kennen, von vornherein abwürgen.

\section{Fazit}

Das Prachtschloss, auf dem in großen Lettern „Interdisziplinarität" prangt, ist voller Türhüter, die einen nicht einlassen oder auf die Ochsentour der Disziplinen lenken. Universitäten, Forschungsförderer und nicht zuletzt die Scientific Community selbst müssten einen Transformationsprozess durchmachen, wenn sich das ändern soll. Lehre und Forschung, die Inter- und Transdisziplinarität einüben und praktizieren, sollten besonders gefördert werden.

\section{Literatur:}

Schneidewind, U. (2012): Die Zürcher Schule für aufgeklärte Nachhaltigkeit. In: GAIA 21, 1/2012. S. 64-65.

Scholz, R. (2011): Environmental literacy in science and society. From knowledge to decisions. New York, Cambridge University Press. WBGU (2011): Welt im Wandel. Gesellschaftsvertrag für eine Große Transformation. Berlin.

\section{AUTOR + KONTAKT}

Claus Leggewie ist Direktor des Kulturwissenschaftlichen Instituts (KWI) in Essen und des Center for Global Cooperation Research in Duisburg. Er ist Mitglied im Wissenschaftlichen Beirat der Bundesregierung Globale Umweltveränderungen (WBGU).

Claus Leggewie, Kulturwissenschaftliches Institut Essen (KWI), Goethestraße 31, 45128 Essen Tel.: +49 201 7204-0, E-Mail: Claus.Leggewie@kwi-nrw.de 
Copyright (C) 2012, IÖW und oekom Verlag. Die Nutzung des Artikels ist Abonnenten von Ökologisches Wirtschaften vorbehalten. Nachdruck und Vervielfältigung des Artikels einschließlich Speicherung und Nutzung auf optischen und elektronischen Datenträgern nur mit Zustimmung der Redaktion von Ökologisches Wirtschaften (http://www.oekologischeswirtschaften.de). 